\title{
LIGNANAS DE Strychnos guianensis (AUBLET) MART.\#
}

\section{Maria Lúcia B. Pinheiro*, Arnaldo F. Imbiriba da Rocha e Marco A. do N. Fernandes}

Departamento de Química, Instituto de Ciências Exatas, Universidade do Amazonas, Av. General Rodrigo Otávio Jordão Ramos, 3000, 69077-000 Manaus - AM

Francisco José Queiroz Monte

Departamento de Química Orgânica e Inorgânica, Centro de Ciências, Universidade Federal do Ceará, CP 12200, 600451-970 Fortaleza - CE

José Daniel Figueroa Villar e Elizabete Rangel Cruz

Instituto Militar de Engenharia, Praça General Tibúrcio, 80, 22290-270 Rio de Janeiro - RJ

Recebido em 7/1/03; aceito em 19/9/03

\begin{abstract}
LIGNANS OF STRYCHNOS GUIANENSIS (AUBLET) MART. The present communication reports the isolation and identification of three lignans from metanolic root extracts of Strychnos guianensis (Aublet) Mart.: olivil (1), cycloolivil (2) and the unknown derivative cycloolivil carbonate (3). From hexane extracts was identified a long chain fatty acid mixture and the triterpene lupeol. The analyses were based on chromatographic and spectroscopy techniques (IR, MS, GC/MS, ${ }^{1} \mathrm{H}-\mathrm{NMR}$ and ${ }^{13} \mathrm{C}-\mathrm{NMR}, 1 \mathrm{D}$ (BB, DEPT 135) and 2D $\left({ }^{1} \mathrm{H},{ }^{1} \mathrm{H}-\mathrm{COSY},{ }^{1} \mathrm{H},{ }^{13} \mathrm{C}-\mathrm{COSY},{ }^{1} \mathrm{H},{ }^{13} \mathrm{C}-\mathrm{COSY}-\mathrm{LR}, \mathrm{HMQC}, \mathrm{HMBC}\right.$ and NOESY) and comparison with literature data.
\end{abstract}

Keywords: Strychnos guianensis; lignans; (+) cycloolivil carbonate.

\section{INTRODUÇÃO}

O gênero Strychnos tem história na medicina folclórica, registrando-se para as espécies sul-americanas, além do uso em curares, efeitos antimaláricos, tônicos, afrodisíacos, febrífugos e anti-anêmicos ${ }^{1}$.

A espécie Strychnos guianensis (Aublet) Mart. apresenta-se como um cipó de porte variável, ocorrendo principalmente no médio e alto Orenoco e por toda a Bacia Amazônica, sendo encontrada em diferentes habitats: terra-firme, várzea, restinga, igapó, mata virgem e secundária $^{2}$. Descrita pela primeira vez em 1783 por Schreber, sob o nome de Toxicaria americana ${ }^{3}$, é considerada uma das espécies mais usadas na preparação do curare, veneno de flecha de algumas tribos de índios da Amazônia. Sabe-se hoje ser ela o famoso "Bejuco de Mavacure", citado por Humboldt no início do século XIX, como um dos principais ingredientes dos curares de Esmeralda, na Venezuela ${ }^{4}$. Estudos químicos recentes sobre esta espécie levaram ao isolamento de alcalóides quaternários ${ }^{5-8}$, justificando o seu freqüente emprego na preparação dos mencionados venenos.

A investigação das ações biológicas de lignanas é relativamente recente, sendo o maior interesse dirigido às ações antitumorais, nas quais os derivados da podofilotoxina (alguns já comercializados) são os mais importantes ${ }^{9,10}$.

Neste trabalho é relatado, principalmente, o isolamento de três lignanas do extrato metanólico do cerne da raiz, (-)-olivil (1), (+)cicloolivil (2) e (+)-carbonato de cicloolivil (3), a última inédita na literatura. Estas lignanas, ao lado de lirioresinol A e B isoladas de Strychnos dinklange $i^{11}$, representam os únicos registros da classe para o gênero, sendo o primeiro relato em espécies de Strychnos americanas. Olivil (1) e cicloolivil (2) têm atividade antioxidante, geralmente associada a atividades anticancerígenas ${ }^{12}$. Do extrato hexânico foi

\section{*e-mail: lbelem@osite.com.br}

\# Dedicado à memória do amigo Prof. Dr. Carlos Humberto Souza Andrade, pela sua grande contribuição ao estudo da fitoquímica brasileira. obtida uma mistura de ácidos graxos, constituída dos ácidos palmítico, oléico, esteárico e nonadecanóico e, a partir do extrato hexânico da casca foi isolado o triterpeno lupeol.<smiles>COc1cc(C2OCC(O)(c3ccc(O)c(OC)c3)C2CO)ccc1O</smiles>

1

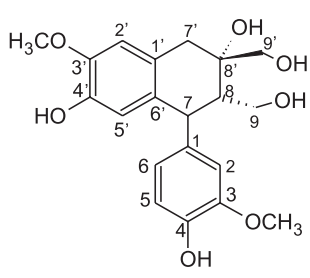

2

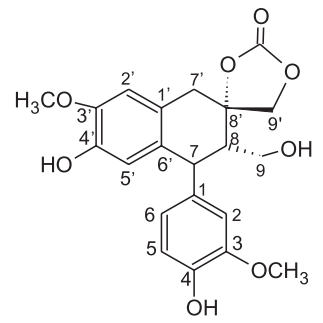

Figura 1. Lignanas de Strychnos guianensis: (-)-olivil (1); (+)-cicloolivil (2); (+)-carbonato de cicloolivil (3)

\section{PARTE EXPERIMENTAL}

\section{Procedimentos gerais}

Os espectros no infravermelho foram obtidos em aparelhos Perkin Elmer, modelos 297 e Spectrum 2000, FT-IR, utilizando pastilhas de $\mathrm{KBr}$. Os espectros de RMN uni e bidimensionais foram registrados em aparelhos Bruker, modelos DPX300 e DRX500, operando, respectivamente, a 300 e $500 \mathrm{MHz}$ para $\mathrm{RMN}$ de ${ }^{1} \mathrm{H}$ e a 75,0 e 125,0 
$\mathrm{MHz}$ para RMN de ${ }^{13} \mathrm{C}$. As amostras foram solubilizadas em $\mathrm{CDCl}_{3}$, $\mathrm{CD}_{3} \mathrm{OD}$ ou $\mathrm{CD}_{3} \mathrm{CN}$ e TMS foi usado como padrão interno. Os espectros de massa foram obtidos através de ionização por impacto de elétrons a 70 eV, em Espectrômetro de Massa de Alta Resolução VG AutoSpec e Espectrômetro de Massa AutoSpec Q. As rotações ópticas foram observadas em polarímetro digital Perkin-Elmer, modelo 341. A determinação de pontos de fusão foi realizada em um bloco de Kofler adaptado a um microscópio. Os valores encontrados não foram corrigidos. A Cromatografia Gasosa (CG) dos ésteres metílicos dos ácidos graxos foi realizada em aparelho Carlo-Erba, modelo 3160, munido com Detector de Ionização de Chama (DIC), utilizando coluna capilar de sílica SE-54, 25 m de comprimento e hélio como gás de arraste, com velocidade $33 \mathrm{~cm} / \mathrm{s}$ a $150^{\circ} \mathrm{C}$. A programação de temperatura foi de $6{ }^{\circ} \mathrm{C} / \mathrm{min}$ até $230{ }^{\circ} \mathrm{C}$ e a temperatura de injeção foi $50{ }^{\circ} \mathrm{C}$. A mistura triterpênica foi analisada por meio de um sistema de Cromatografia Gasosa de Alta Resolução (CGAR-EM) Hewlett Packard modelo 6890, com analisador de íons quadrupolo e ionização por impacto de elétrons, $70 \mathrm{eV}$, usando coluna capilar HP-5 (5\% fenil e $95 \%$ dimetilsiloxano), $30 \mathrm{~m}$ x 0,32 mm. A identificação foi feita por meio de uma biblioteca de espectros de massas (Wiley 59943 B).

\section{Material botânico}

A identificação botânica de $S$. guianensis (Aublet) Mart. foi feita pelo Dr. A. J. M. Leewenberg, do Departamento de Taxonomia de Plantas da Universidade de Agricultura de Wageningen, Holanda. As exsicatas encontram-se no Herbário do Instituto Nacional de Pesquisas da Amazônia (INPA), registradas sob número 150.295.

\section{Extração e isolamento}

\section{Cerne da raiz}

800,0 g de material botânico seco e moído foram extraídos durante 48 h com hexano em aparelho Soxhlet, obtendo-se após concentração à pressão reduzida, um resíduo $\mathrm{A}(7,5 \mathrm{~g})$. A torta remanescente foi macerada em uma mistura de metanol: ácido acético (99:1). Após concentração do extrato à pressão reduzida, acrescentou-se 150 $\mathrm{mL}$ de uma solução de ácido acético a 5\% e extraiu-se com clorofórmio. A partir da fração clorofórmica foi obtida uma pasta marrom B $(0,428 \mathrm{~g})$ que, aplicada em coluna de sílica gel utilizando como fase móvel, hexano, hexano/clorofórmio, clorofórmio, clorofórmio/ metanol e metanol, em variados gradientes de eluição, forneceu 0,037 $\mathrm{g}$ de um sólido que foi identificado como o (+) carbonato de cicloolivil (3). A fração aquosa ácida foi alcalinizada com carbonato de sódio até $\mathrm{pH} 8$ e submetida à nova extração com clorofórmio. Esta fração, tratada com sulfato de sódio anidro e concentrada em evaporador rotatório, à pressão reduzida, forneceu uma pasta escura C $(0,615 \mathrm{~g})$. A fração aquosa básica foi levada a pH 11 com hidróxido de sódio e novamente extraída com clorofórmio obtendo-se, após evaporação do solvente, um resíduo marrom D (0,200 g). Pela semelhança revelada em cromatografia de camada delgada (CCD) analítica, C e D foram reunidos e cromatografados como $\mathrm{B}$, fornecendo duas frações com sólidos cristalinos. Estes foram purificados através de recristalizações sucessivas com mistura de clorofórmio, metanol e hexano (1:5:1) e identificados, respectivamente, como (-)-olivil (1) $(0,040 \mathrm{~g})$ e $(+)$ - cicloolivil (2) $(0,020 \mathrm{~g})$. As duas colunas cromatográficas forneceram frações alcaloídicas complexas que não foram purificadas pela metodologia utilizada.

\section{Saponificação de A}

Foi realizada segundo técnicas convencionais ${ }^{13}$, fornecendo 1,48 g de óleo contendo a mistura de ácidos graxos A1.

\section{Metilação de $\mathrm{Al}^{14}$}

Foram colocados $0,450 \mathrm{~g}$ de $\mathrm{A} 1 \mathrm{em}$ um tubo de ensaio e 4,0 mL de solução de hidróxido de sódio em metanol $0,5 \mathrm{~mol} \mathrm{~L}^{-1}$. O tubo foi fechado, agitado e aquecido em banho-maria até dissolver todos os glóbulos gordurosos (3-5 min). Após resfriamento sob água corrente, adicionou-se $5,0 \mathrm{~mL}$ do reagente esterificante $(20 \%$ de fluoreto de boro em metanol), agitando e levando a aquecimento e resfriamento, como anteriormente. Posteriormente acrescentou-se 4,0 mL de solução aquosa saturada de cloreto de sódio e 5,0 mL de hexano. A fase hexânica foi analisada por cromatografia gasosa e espectroscopia no IV, revelando a presença de ésteres metílicos dos ácidos: palmítico (44\%), oléico (33\%), esteárico (6\%) e ácido nonadecanóico $(7 \%)$.

\section{Casca da raiz}

20,0 g de casca da raiz seca e moída foram submetidos à extração com hexano em Soxhlet, por um período de 48 h. Após evaporação do solvente, foi obtido um resíduo amarelado, que submetido a sucessivas recristalizações em clorofórmio/metanol forneceu um sólido cristalino identificado como o triterpeno lupeol (0,600 g).

(-)-Olivil (1) - sólido, p.f. $144-146{ }^{\circ} \mathrm{C} ;[\alpha]_{\mathrm{D}}^{25}-46,0$ (c 0,12, $\mathrm{MeOH})$; I.V. (KBr, $\left.v_{\max } \mathrm{cm}^{-1}\right)-3400,2910,2830,1600,1510,1445$, 1330, 1270, 1240, 1150, 1120, 1090, 1030, 970, 940, 860, 820, 770, 750, 700. EMAR, IE, $70 \mathrm{eV}, \mathrm{m} / \mathrm{z}(\%)-376,15228(21)\left[\mathrm{M}^{+*}\right]$, 238,08364 (6), 196,07302 (9), 180,07887 (15), 163,07563 (10), 138,67759 (97), 137,06033 (100). RMN de ${ }^{1} \mathrm{H}$ e RMN de ${ }^{13} \mathrm{C}$ (Tabela 1$)$.

(+)-Cicloolivil (2) - sólido, p.f. $162-166^{\circ} \mathrm{C} ;[\alpha]_{\mathrm{D}}^{25}+39,5$ (c 0,12, $\mathrm{MeOH})$; I.V. $\left(\mathrm{KBr}, v_{\text {max }} \mathrm{cm}^{-1}\right)$ - 3420, 3310, 2930, 2910, 1600, 1520, 1450, 1370, 1260, 1220, 1150, 1130, 1030, 890, 860, 840, 820, 780. E.M., IE, 70 eV (\%) 376 (31) [ $\left.\mathrm{M}^{+*}\right], 358$ (13), 340 (9), 327 (54), 309 (34), 297 (100), 284 (7), 265 (15), 191 (28), 137 (31), 115 (6). RMN de ${ }^{1} \mathrm{H}$ e RMN de ${ }^{13} \mathrm{C}$ (Tabela 2$)$.

(-)-Carbonato de cicloolivil (3) - sólido, p.f. 118-120 ${ }^{\circ} \mathrm{C} ;[\alpha]^{25}+$ 68,0 (c 0,05, MeOH); I.V. (KBr, $\left.v_{\max } \mathrm{cm}^{-1}\right) 3380,1820,1785,1600$, 1510, 1450, 1430, 1365, 1250, 1210, 1030, 760. EMAR, IE $70 \mathrm{eV}$ $\mathrm{m} / \mathrm{z}(\%) 402,12992(21)\left[\mathrm{M}^{+*}\right], 353,10223$ (14), 309,11198 (100), 297,11242 (14), 241,08631 (12), 187,07591 (9), 137,06016 (17), 115,05471 (8), 77,03897 (6). RMN de ${ }^{1} \mathrm{H}$ e RMN de ${ }^{13} \mathrm{C}$ (Tabela 3).

Lupeol, sólido p.f. $178-180^{\circ} \mathrm{C}$. I.V. $\left(\mathrm{KBr}, v_{\max } \mathrm{cm}^{-1}\right)$ : 3313,3068 , 2946, 1640, 1458, 1381, 1302, 1190, 1141, 1106, 1038, 981, 944,

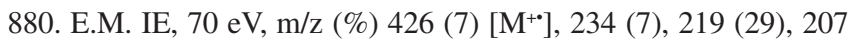
(44) 189 (59)135 (69) 121 (80), 107 (100). RMN de ${ }^{1} \mathrm{H}, 500 \mathrm{MHz}$, $\mathrm{CDCl}_{3}, \delta: 4,54\left(1 \mathrm{H}, \mathrm{d}, 2,0 \mathrm{~Hz}, \mathrm{H}_{\mathrm{A}}-29\right) 4,67\left(1 \mathrm{H}, \mathrm{d}, 2,0 \mathrm{~Hz}, \mathrm{H}_{\mathrm{B}}-29\right)$ 3,16 (1H, dd, 11,4 e 4,9 Hz, H-3), 2,34 (2H, m, H-19), 1,68 (3H, s, H-30), 1,01, 0,97, 0,95, 0,81, 0,77, 0,74 (todos 3H, s, H-23 a H-28). RMN de ${ }^{13} \mathrm{C}, 125 \mathrm{MHz}, \mathrm{CDCl}_{3}, \delta: 38,78$ (C-1), 27,78 (C-2), 79,05 (C-3), 38,91 (C-4); 55,37 (C-5), 18,36 (C-6), 34,36 (C-7), 40,91 (C8), 50,51 (C-9), 37,26 (C-10), 20,99 (C-11), 25,22 (C-12), 38,13 (C-13), 42,90 (C-14), 27,51 (C-15), 35,64 (C-16); 43,04 (C-17), 48,38 (C-18), 48,03 (C-19), 150,96 (C-20), 29,92 (C-21), 40,06 (C-22), 28,04 (C-23), 15,40 (C-24), 16,15 (C-25), 16,03 (C-26), 14,60 (C27), 18,05 (C-28), 109,36 (C-29), 19,36 (C-30).

\section{RESULTADOS E DISCUSSÃO}

\section{Identificação e determinação estrutural de 1, 2 e 3}

Os espectros na região do infravermelho de 1, 2 e 3 mostraram uma grande semelhança em torno de $3400 \mathrm{~cm}^{-1}$, característica de grupamentos $\mathrm{OH}$, e três bandas entre 1600 e $1450 \mathrm{~cm}^{-1}$, acompanhadas de absorções na faixa de $890-740 \mathrm{~cm}^{-1}$, revelando tratar-se de substância aromática. Somente $\mathbf{3}$ evidenciou presença de carbonila, 
com absorções em $1785 \mathrm{~cm}^{-1}$ e $1820 \mathrm{~cm}^{-1}$ (ombro). Por intermédio dos espectros de RMN de ${ }^{13} \mathrm{C}$ (BB e DEPT-135) verificou-se a presença de vinte sinais de carbonos para os compostos $\mathbf{1}$ e $\mathbf{2}$, sendo registrado um sinal adicional para 3 em $\delta$ 155,95, atribuído a um grupo carbonila. Todos os compostos apresentaram sinais típicos para dois grupos metoxilas em torno de $\delta 56,00$. Os espectros de massa de alta resolução (EMAR) registraram íon molecular com razão de massa/carga $(\mathrm{m} / \mathrm{z}) 376,15228$, compatível com fórmula molecular $\mathrm{C}_{20} \mathrm{H}_{24} \mathrm{O}_{7}$ para $1 \mathrm{e}, 402,12992$ para 3, correspondendo à fórmula $\mathrm{C}_{21} \mathrm{H}_{22} \mathrm{O}_{8}$. O espectro de massa de baixa resolução de $\mathbf{2}$ registrou íon molecular com razão de massa/carga $(\mathrm{m} / \mathrm{z}) 376$, levando à mesma fórmula molecular de 1. Este conjunto de dados espectrais sugeriu que se tratavam de substâncias com esqueleto básico constituído de 18 átomos de carbono, de natureza fenilpropanóide $\left(\mathrm{C}_{6}-\mathrm{C}_{3}\right)_{2}$, provavelmente lignanas. Por meio dos espectros de RMN de ${ }^{1} \mathrm{H}$ e RMN de ${ }^{13} \mathrm{C}$ (Tabela 1), experiências de dupla ressonância em frequiências selecionadas e comparação com dados da literatura ${ }^{15}$ a estrutura $\mathbf{1}$ foi identificada como a lignana tetraidrofurânica (-)-olivil (1). A presença de (+)-cicloolivil (2) foi estabelecida com base na análise de seus espectros, principalmente de Ressonância Magnética Nuclear $1 \mathrm{D}$ e 2D, sendo realizadas atribuições inequívocas de deslocamentos químicos através de técnicas de detecção inversa (HMQC e HMBC) e NOESY (Tabela 2). Isto foi necessário em vista das atribuições dos deslocamentos químicos discrepantes observados na literatura para os metilenos carbinólicos 9- $\mathrm{CH}_{2} \mathrm{OH}$ e 9'- $\mathrm{CH}_{2} \mathrm{OH}$. Shogomu-Tih et $a l .{ }^{16}$ atribuíram os valores $\delta 69,5(\mathrm{C}-9)$ e $\delta 61,0$ (C'-9), enquanto Abe et al..$^{15}$, Agraw e Pathak ${ }^{17}$ e os dados obtidos no presente trabalho indicam que os valores foram trocados no primeiro registro.

Para a determinação estrutural de $\mathbf{3}$ tomou-se, como modelo, os dados espectrais do (+) cicloolivil (2). Observou-se que 3 apresentava sinais para cinco hidrogênios entre $\delta$ 6,8-6,0 distribuídos em dois conjuntos, compatíveis com a presença de dois anéis aromáticos. $\mathrm{O}$ primeiro, 1,3,4-trissubstituído, apresentou sinais em $\delta$ 6,60 (dd, 8,5 e $2,0 \mathrm{~Hz}, 1 \mathrm{H})$ acoplado com sinais em $\delta 6,75(\mathrm{~d}, 2,0 \mathrm{~Hz}, 1 \mathrm{H})$ e $\delta 6,76$ (d, 8,5 Hz, 1H), e o segundo, 1,2,4,5-tetrassubstituído, revelou dois singletos em $\delta 6,16$ e $\delta 6,65$. Estes dados estavam de acordo com os espectros de RMN de ${ }^{13} \mathrm{C}$ (BB, DEPT-135) e ${ }^{1} \mathrm{H},{ }^{13} \mathrm{C}-\mathrm{COSY}$ que evidenciaram a presença de cinco $\mathrm{CH}\left(\mathrm{sp}^{2}\right)$ e oito carbonos não hidrogenados, dos quais quatro poderiam estar ligados a oxigênio. Os segmentos propanoídicos foram identificados por meio das mesmas técnicas. Um segmento ( $\mathrm{CHCHCHO})$, pelos sinais em $\delta 45,13$ (C-7), $\delta 49,53$ (C-8) e $\delta$ 60,05 (C-9), correspondentes, respectivamente, aos sinais em $\delta 3,93(\mathrm{~d}, 11,0 \mathrm{~Hz}, 1 \mathrm{H}), \delta 2,20$ (ddd, 11,0, 6,0 e $3,0 \mathrm{~Hz}, 1 \mathrm{H})$ e $\delta 3,63(\mathrm{~m}, 1 \mathrm{H}) / 3,50(\mathrm{~m}, 1 \mathrm{H})$ do espectro de $\mathrm{RMN}$ de ${ }^{1} \mathrm{H}$. O outro $\left(\mathrm{CH}_{2} \mathrm{C}(\mathrm{O}) \mathrm{CH}_{2} \mathrm{O}\right)$, pelo deslocamento químico de um carbono não hidrogenado em $\delta 84,84\left(C^{\prime}-8\right)$ no espectro de RMN de ${ }^{13} \mathrm{C}$ (BB), e os sinais em $\delta 40,64$ (C-7') e $\delta 73,44$ (C-9') correlacionados separadamente, no espectro ${ }^{1} \mathrm{H},{ }^{13} \mathrm{C}-\mathrm{COSY}$, a dupletos em $\delta 3,27(16,70 \mathrm{~Hz}, 1 \mathrm{H}) / \delta 3,05(16,70 \mathrm{~Hz}, 1 \mathrm{H})$ e $\delta 4,18(8,8 \mathrm{~Hz}, 1 \mathrm{H}) /$ $\delta 4,87(8,8 \mathrm{~Hz}, 1 \mathrm{H})$. A junção das sub-estruturas evidenciadas teve como base a análise do espectro ${ }^{1} \mathrm{H},{ }^{13} \mathrm{C}$-COSY-LR (Tabela 3) onde se verificou, entre outros, os acoplamentos de H-9' A com C-8, C-8' e C-7', H-7 com C-1' e C-6', H-5' com C-7, configurando a estrutura de uma lignana com esqueleto do tipo tetraidronaftaleno. Por intermédio do espectro ${ }^{1} \mathrm{H},{ }^{13} \mathrm{C}$-COSY-LR também foi possível localizar inequivocamente duas hidroxilas em C-4 e C-4', restando as posições C-3 e C-3' para as duas metoxilas, confirmadas com base nos deslocamentos químicos dos respectivos carbonos. O acréscimo de 26 Da no íon molecular de $\mathbf{3}$ em relação ao de $\mathbf{2}$ nos espectros de massa, juntamente com as absorções na região do infravermelho ${ }^{18}$ em 1785 e $1820 \mathrm{~cm}^{-1}$, e o deslocamento químico no espectro de RMN de ${ }^{13} \mathrm{C}$ em $\delta 155,95$ evidenciaram a presença do grupo carbonila de um cicloalquilcarbonato derivado do cicloolivil (2). Os sinais dos hidrogênios geminais em $\delta 4,18(\mathrm{~d}, 8,8 \mathrm{~Hz}, 1 \mathrm{H})$ e $\delta 4,87$ (d, 8,8 Hz, $1 \mathrm{H}$ ), correlacionados ao carbono metilênico em $\delta 73,44$ (C-9'), e o deslocamento químico em $\delta 84,84$ (C-8') indicaram que se tratava

Tabela 1. Dados espectrais de $\mathrm{RMN}$ de ${ }^{1} \mathrm{H}^{*}(300 \mathrm{MHz})$ e ${ }^{13} \mathrm{C}(75 \mathrm{MHz})$ de $\mathbf{1}$ e de olivil ${ }^{15}, \delta(\mathrm{ppm})$

\begin{tabular}{|c|c|c|c|c|}
\hline \multirow[b]{2}{*}{ Posição } & \multicolumn{2}{|c|}{1 , solvente acetonitrila- $\mathrm{d}_{3}$} & \multicolumn{2}{|c|}{ Olivil, solvente piridina $-\mathrm{d}_{5}$} \\
\hline & $\delta_{\mathrm{H}}$ & $\delta_{\mathrm{C}}$ & $\delta_{\mathrm{H}}$ & $\delta_{\mathrm{C}}$ \\
\hline $1^{\prime}$ & & 129,4 & & 130,1 \\
\hline 2 ' & $7,02(\mathrm{~d}, 1,8 ; 1 \mathrm{H})$ & 113,9 & $7,32(\mathrm{~d}, 2,0 ; 1 \mathrm{H})$ & 115,4 \\
\hline $3^{\prime}$ & & 146,7 & & 148,2 \\
\hline $4^{\prime}$ & $6,89(\mathrm{~s}, \mathrm{OH})$ & 144,6 & & 146,7 \\
\hline 5 , & $6,91(\mathrm{~d}, 7,7 ; 1 \mathrm{H})$ & 114,2 & $7,22(\mathrm{~d}, 8,0 ; 1 \mathrm{H})$ & 116,0 \\
\hline $6^{\prime}$ & $6,89(\mathrm{dd}, 7,7 ; 1,8 ; 1 \mathrm{H})$ & 122,8 & $7,19(\mathrm{dd}, 8,0 ; 2,0 ; 1 \mathrm{H})$ & 123,7 \\
\hline \multirow[t]{2}{*}{7} & A $3,01(\mathrm{~d}, 14,0 ; 1 \mathrm{H})$ & 39,9 & $3,41(\mathrm{~d}, 14,0 ; 1 \mathrm{H})$ & 40,7 \\
\hline & B $3,10(\mathrm{~d}, 14,0 ; 1 \mathrm{H})$ & & $3,57(\mathrm{~d}, 14,0 ; 1 \mathrm{H})$ & \\
\hline $8^{\prime}$ & $3,25(\mathrm{~s}, \mathrm{OH})$ & 81,2 & & 82,0 \\
\hline \multirow[t]{2}{*}{$9^{\prime}$} & A $3,68(\mathrm{~d}, 9,1 ; 1 \mathrm{H})$ & 76,6 & $4,24(\mathrm{~d}, 9,0 ; 1 \mathrm{H})$ & 78,1 \\
\hline & B $3,92(d, 9,1 ; 1 \mathrm{H})$ & & $4,35(\mathrm{~d}, 9,0 ; 1 \mathrm{H})$ & \\
\hline 1 & & 134,8 & & 135,6 \\
\hline 2 & $7,22(\mathrm{~d}, 2,0 ; 1 \mathrm{H})$ & 110,2 & $7,62(\mathrm{~d}, 2,0 ; 1 \mathrm{H})$ & 111,6 \\
\hline 3 & & 147,1 & & 148,7 \\
\hline 4 & $6,89(\mathrm{~s}, \mathrm{OH})$ & 145,4 & & 147,6 \\
\hline 5 & $6,91(\mathrm{~d}, 7,7 ; 1 \mathrm{H})$ & 114,2 & $7,22(\mathrm{~d}, 8,0 ; 1 \mathrm{H})$ & 116,1 \\
\hline 6 & $7,00(\mathrm{dd}, 7,7 ; 2,0 ; 1 \mathrm{H})$ & 119,3 & $7,36(\mathrm{dd}, 8,0 ; 2,0 ; 1 \mathrm{H})$ & 120,5 \\
\hline 7 & $4,78(\mathrm{~d}, 7,0 ; 1 \mathrm{H})$ & 83,7 & $5,33(\mathrm{~d}, 7,0 ; 1 \mathrm{H})$ & 84,8 \\
\hline 8 & $2,39(\mathrm{dd}, 7,0 ; 7,0 ; 1 \mathrm{H})$ & 60,2 & $3,02(\mathrm{~m})$ & 62,1 \\
\hline \multirow[t]{3}{*}{9} & A $3,78(\mathrm{dd}, 10,7 ; 7,0 ; 1 \mathrm{H})$ & 59,6 & $4,2-4.3$ & 60,5 \\
\hline & B $3,82(\mathrm{dd}, 10,7 ; 7,0 ; 1 \mathrm{H})$ & & & \\
\hline & $3,25(\mathrm{~s}, \mathrm{OH})$ & & & \\
\hline $2 \mathrm{CH}_{3} \mathrm{O}$ & $3,98(\mathrm{~s}, 6 \mathrm{H})$ & 55,5 & $3,67(\mathrm{~s}, 3 \mathrm{H})$ e $3,72(\mathrm{~s}, 3 \mathrm{H})$ & 55,8 e 55,9 \\
\hline
\end{tabular}

\footnotetext{
* Multiplicidades e constantes de acoplamento $(J)$ em Hz., entre parênteses; $\delta$ (ppm). Reproduzida da ref. 15, com permissão da Elsevier
} 
Tabela 2. Dados espectrais de RMN de ${ }^{1} \mathrm{H}(500 \mathrm{MHz})$ e $\mathrm{RMN}$ de ${ }^{13} \mathrm{C}(125 \mathrm{MHz})$ de 2 em $\mathrm{CD}_{3} \mathrm{OD}, \delta$ (ppm)

\begin{tabular}{|c|c|c|c|c|}
\hline \multirow[b]{2}{*}{ Posição } & \multicolumn{2}{|c|}{$\operatorname{HMQC}\left({ }^{1} J_{\mathrm{CH}}\right)^{\mathrm{a}}$} & \multirow{2}{*}{$\begin{array}{l}\text { NOESY }{ }^{\mathrm{b}} \\
\text { Número do } H\end{array}$} & \multirow{2}{*}{$\begin{array}{c}\mathrm{HMBC}^{\mathrm{c}} \\
\text { Número do C }\end{array}$} \\
\hline & $\delta_{\mathrm{H}}$ & $\delta_{\mathrm{C}}$ & & \\
\hline 1 ' & \multirow{4}{*}{$6,61(\mathrm{~s}, 1 \mathrm{H})$} & 133,60 & \multirow{4}{*}{ 7'A } & \multirow{4}{*}{ 1'; 6’; 4', 7' } \\
\hline $2^{\prime}$ & & 113,08 & & \\
\hline 3 ' & & 147,50 & & \\
\hline 4' & & 145,33 & & \\
\hline 5 , & $6,16(\mathrm{~s}, 1 \mathrm{H})$ & 117,36 & 7 & 6’; 3’; 7 \\
\hline 6 & - & 126,46 & & \\
\hline \multirow[t]{2}{*}{ 7' } & A $2,59(\mathrm{~d}, 16,72,1 \mathrm{H})$ & 39,95 & 2' & $8^{\prime} ; 9^{\prime} ; 8 ; 2^{\prime} ; 6^{\prime} ; 1^{\prime}$ \\
\hline & B $3,19(\mathrm{~d}, 16,72,1 \mathrm{H})$ & & 8 & 8’; 2’; 6’; 1' \\
\hline 8 ' & & 74,95 & & \\
\hline \multirow[t]{2}{*}{9 , } & A $3,56(\mathrm{dd}, 11,14,1 \mathrm{H})$ & 69,45 & \multirow{6}{*}{8} & 7'; 8 \\
\hline & B $3,77(\mathrm{~d}, 11,14,1 \mathrm{H})$ & & & $7 ; 88$ \\
\hline 1 & & 138,49 & & \multirow{4}{*}{$6 ; 4 ; 1$} \\
\hline 2 & $6,67(\mathrm{~d}, 1,6,1 \mathrm{H})$ & 114,07 & & \\
\hline 3 & & 149,13 & & \\
\hline 4 & & 146,12 & & \\
\hline 5 & $6,75(\mathrm{~d}, 8,00,1 \mathrm{H})$ & 116,06 & & $1 ; 4 ; 3$ \\
\hline 6 & $6,65(\mathrm{dd}, 8,0 ; 1,6,1 \mathrm{H})$ & 123,58 & & 7,2 \\
\hline 7 & $4,00(\mathrm{~d}, 11,5,1 \mathrm{H})$ & 44,91 & $5^{\prime}, 6,2$ & $2 ; 6 ; 1 ; 11$ \\
\hline 8 & $2,01(\mathrm{dt}, 11,5 ; 2,6,1 \mathrm{H})$ & 47,67 & 7’B, 9’B, 6, 2 & \\
\hline \multirow[t]{2}{*}{9} & A $3,54(\mathrm{dd}, 10,4 ; 4,01,1 \mathrm{H})$ & 60,88 & & $8 ; 8$ \\
\hline & В $3,79(\mathrm{dd}, 10,4 ; 2,6,1 \mathrm{H})$ & & & $7 ; 8$ \\
\hline $3-\mathrm{OCH}_{3}$ & $3,75(\mathrm{~s}, 3 \mathrm{H})$ & 56,43 & 2 & 3 \\
\hline $3^{\prime}-\mathrm{OCH}_{3}$ & $3,78(\mathrm{~s}, 3 \mathrm{H})$ & 56,46 & $2^{\prime}$ & $3^{\prime}$ \\
\hline
\end{tabular}

${ }^{a}$ Multiplicidades e constantes de acoplamento $(J)$ em Hz, entre parênteses; ${ }^{\mathrm{b}}$ correlações selecionadas de H ao hidrogênio indicado; ${ }^{\mathrm{c}}$ correlações selecionadas de $\mathrm{H}$ ao carbono indicado.

Tabela 3. Dados espectrais de $\mathrm{RMN}$ de ${ }^{1} \mathrm{H}^{*}(500 \mathrm{MHz})$ e ${ }^{13} \mathrm{C}(75$ $\mathrm{MHz})$ de $3 \mathrm{em} \mathrm{CD}{ }_{3} \mathrm{CN}, \delta(\mathrm{ppm})$

\begin{tabular}{|c|c|c|c|}
\hline Posição & $\begin{array}{c}{ }^{1} \mathrm{H},{ }^{13} \mathrm{C}-\operatorname{COSY}\left({ }^{1} J_{\mathrm{CH}}\right) \\
\delta_{\mathrm{H}}\end{array}$ & $\delta_{\mathrm{C}}$ & $\begin{array}{c}{ }^{1} \mathrm{H},{ }^{13} \mathrm{C}-\mathrm{COSY}-\mathrm{LR}^{\mathrm{a}} \\
\text { Número do } C\end{array}$ \\
\hline $1 '$ & & 132,13 & \\
\hline $2^{\prime}$ & $6,65(\mathrm{~s}, 1 \mathrm{H})$ & 112,12 & 3'; 1'; 7' \\
\hline $3^{\prime}$ & & 147,00 & \\
\hline $4^{\prime}$ & $6,24(\mathrm{~s}, \mathrm{OH})$ & 145,72 & 5 \\
\hline $5^{\prime}$ & $6,16(\mathrm{~s}, 1 \mathrm{H})$ & 116,58 & 3’; 4'; 6’; 7; 7' \\
\hline $6^{\prime}$ & & 124,17 & \\
\hline 7 ' & $\begin{array}{l}\text { A } 3,05(\mathrm{~d}, 16,7 ; 1 \mathrm{H}) \\
\text { B } 3,27(\mathrm{~d}, 16,7 ; 1 \mathrm{H})\end{array}$ & 40,64 & $1^{\prime} ; 6^{\prime} ; 8^{\prime} ; 9^{\prime} ; 8$ \\
\hline $8^{\prime}$ & & 84,84 & \\
\hline $9^{\prime}$ & $\begin{array}{l}\text { A } 4,18(\mathrm{~d}, 8,8 ; 1 \mathrm{H}) \\
\text { B } 4,87(\mathrm{~d}, 8,8 ; 1 \mathrm{H})\end{array}$ & 73,44 & $8^{\prime} ; 8 ; 7^{\prime}$ \\
\hline 1 & & 137,51 & \\
\hline 2 & $6,75(\mathrm{~d}, 2,1 \mathrm{H})$ & 113,62 & $3 ; 4 ; 1 ; 6$ \\
\hline 3 & & 148,26 & \\
\hline 4 & $6,42(\mathrm{~s}, \mathrm{OH})$ & 145,63 & 5 \\
\hline 5 & $6,76(\mathrm{~d}, 8,5 ; 1 \mathrm{H})$ & 115,61 & $3 ; 4 ; 1 ; 6$ \\
\hline 6 & $6,60(\mathrm{dd}, 8,5$ e $2,01 \mathrm{H})$ & 122,64 & $4 ; 2$ \\
\hline 7 & $3,93(\mathrm{~d}, 11,0 ; 1 \mathrm{H})$ & 45,13 & $\begin{array}{l}1 ; 1^{\prime} ; 6 ’ ; 6 ; 2 ; \\
9 ; 8\end{array}$ \\
\hline 8 & $\begin{array}{l}2,20(\mathrm{ddd}, 11,0 ; 6,0 \\
3,0 ; 1 \mathrm{H})\end{array}$ & 49,53 & \\
\hline 9 & $\begin{array}{l}\text { A } 3,50(\mathrm{~m}) \\
\text { B } 3,63(\mathrm{~m}) \\
2,81(\mathrm{t}, 4,5, \mathrm{OH})\end{array}$ & 60,05 & \\
\hline $\mathrm{OCH}_{3}$ & $3,78(\mathrm{~s}, 3 \mathrm{H})$ & 56,57 & \\
\hline $\mathrm{OCH}_{3}$ & $3,80(\mathrm{~s}, 3 \mathrm{H})$ & $\begin{array}{l}56,62 \\
15505\end{array}$ & \\
\hline $\mathrm{O}-\mathrm{CO}-$ & & 155,95 & \\
\hline
\end{tabular}

* Multiplicidades e constantes de acoplamento $(J)$ em Hz, entre parênteses; ${ }^{\text {a }}$ correlações selecionadas de $\mathrm{H}$ ao carbono indicado. de um cicloalquilcarbonato de 5 membros, conforme mostrado na estrutura 3.

\section{Identificação do lupeol}

O triterpeno lupeol foi identificado através de métodos cromatográficos e espectrométricos (CG/EM, IV e RMN) e comparação com dados da literatura ${ }^{19}$, principalmente $\mathrm{RMN} \mathrm{de}{ }^{13} \mathrm{C}$.

\section{Identificação da mistura de ácidos graxos}

Após saponificação seguida de acidificação do material graxo A, proveniente da marcha química utilizada, foi obtido um material oleoso, cujo espectro no IV apresentou bandas intensas em 2920 e 2880 $\mathrm{cm}^{-1}$ superpostas a uma absorção larga entre $3500-2800 \mathrm{~cm}^{-1}$, compatíveis, respectivamente, com estiramentos de grupos $\mathrm{CH}$ e $\mathrm{OH}$. Absorção adicional em $1715 \mathrm{~cm}^{-1}$, caracterizou a presença de carboxila típica de uma mistura de ácidos graxos. Após metilação, com $\mathrm{BF}_{3} / \mathrm{MeOH}$, foi confirmado o processo de esterificação pelo desaparecimento da absorção referente à hidroxila e deslocamento da absorção da carbonila para freqüência mais alta $\left(1750 \mathrm{~cm}^{-1}\right)$. Por intermédio de análise em cromatografia gasosa foi identificada, baseada nos tempos de retenção e co-injeção com amostras autênticas, uma mistura de ésteres: palmitato de metila (44\%), oleato de metila (33\%), estearato de metila (6\%) e nonadecanoato de metila (7\%).

\section{CONSIDERAÇÕES FINAIS}

O carbonato de cicloolivil (3) pode ter sido formado pela reação do cicloolivil (2) com o carbonato de sódio usado na marcha química. Entretanto, tentativas para obter 3 a partir do cicloolivil (2), simulando as condições de isolamento, foram infrutíferas. Assim, não se descarta a hipótese do carbonato de cicloolivil ser um produto 
natural, pois é sabido que, apesar de raro, há registros da presença de carbonatos de alquila naturais na literatura ${ }^{20}$.

Os estudos fitoquímicos do gênero Strychnos têm se concentrado na obtenção de alcalóides, todavia, a potencialidade farmacológica dos lignóides estimula uma investigação mais intensiva do conteúdo não alcaloídico deste gênero, quiçá, responsável por muitas das virtudes que lhe são atribuídas pela medicina popular.

\section{AGRADECIMENTOS}

Ao CNPq pela bolsa de doutoramento concedida à M. L. Belém Pinheiro. Ao Dr. E. Silveira e D. E. de Andrade (CENAUREMNUFC) pelos espectros de RMN. Ao pesquisador I. R. Luz (INPA) pelos cromatogramas dos ésteres metílicos dos ácidos graxos. À FIOCRUZ pelo CG/EM. Ao Dr. A. J. M. Leewenberg, do Departamento de Taxonomia de Plantas da Universidade de Agricultura de Wageningen, Holanda pela identificação botânica.

\section{REFERÊNCIAS}

1. Quetin-Leclercq, J.; Angenot, L.; Bisset, N. G.; J. Ethnopharmacol. 1990, 28,1 .

2. Krukoff, B. A.; Lloydia 1972, 35, 193.

3. Ducke, A.; Boletim Técnico do Instituto Agronômico do Norte 1965, 30, 1.

4. Bettolo, G. B.; Bisset; N. G.; Lloydia 1972, 35, 195.
5. Quetin- Leclercq, J.; Warin, R.; Belém-Pinheiro, M. L.; Mavar-Manga, H.; Angenot, L.; Phytochemistry 1995, 40, 1557.

6. Penelle, J.; Tits, M.; Christen, P.; Brandt, V.; Frederich, M.; Angenot, L.; J. Nat. Prod. 1998, 62, 898 .

7. Penelle, J.; Tits, M.; Christen, P.; Molgó, J.; Brandt, V.; Frederich, M.; Angenot, L.; Phytochemistry 2000, 53, 1057.

8. Penelle, J.; Christen, P.; Molgó, J.; Tits, M.; Brandt, V.; Frederich, M.; Angenot, L.; Phytochemistry 2001, 58, 619.

9. Yoshida, M.; Gottlieb, O. R.; Quim. Nova 1984, 7, 250.

10. Ward, R. S.; Nat. Prod. Rep. 1997, 14, 43.

11. Michel, S.; Tillequim, F.; Koch, M.; Assi, L. A.; J. Nat. Prod. 1982, 45, 489

12. Lee, S. K.; Mbwambo, Z. H.; Chung, H.; Luyengi, L.; Gamez, E. J., Mehta; R. G.; Kimghom, A. D.; Pezzuto, J. M.; Comb. Chem \& High Throughput Screen. 1998, 1, 35.

13. Matos, F. J. A.; Introdução à Fitoquímica Experimental, $2^{\mathrm{a}}$ ed., UFC: Fortaleza, 1998, p. 67

14. Maia, E. L.; Amaya, D. B. R.; Rev. Inst. Adolfo Lutz 1993, 53, 27.

15. Abe, F.; Yamauchi, T.; Wan, A. S.; Phytochemistry 1989, 28, 3473.

16. Shogomu-Tih, R.; Bodo, B.; Niasse, B.; Sondenga, B. L.; Planta Med. 1985, 464.

17. Agrawl, P. K.; Pathak, A. K.; Magn. Reson. Chem. 1994, 32, 753.

18. Colthup, N. B.; Daly, L. H.; Wiberley, S. E.; Introduction to Infrared and Raman Spectroscopy, Academic Press: New York, 1964, p. 255.

19. Patra, A.; Mukhopadhyay, A.; Mitra, A. K.; Org. Magn. Reson. 1981, 17, 166.

20. Lima, M. P.; Tese de Doutorado, Universidade Federal de São Carlos, Brasil, 2000. 